\section{- Redes De Mejora de la Calidad}

(1) Juan Ceval I os A.

\section{RESUMEN}

El artículo trata sobre la necesidad de herramientas con mayor potencia para la mejora de la calidad. Se utiliza la combinación, de manera creativa, de la Filosofía del Mejoramiento Continuo, la Teoría General de Sistemas y la Teoría General de Redes, a fin de desarrollar las Redes de Mejora de la Calidad que permitan optimizar los sistemas y procesos en las organizaciones.

Palabras Claves: Calidad. Redes. Sistemas. Mejoramiento Continuo.

NETWORKS OF QuALITY IMPROVEMENT ABSTRACT

This article deals about the need of better highly-powered tools for quality improvement. A creative combination of Continuous Improvement Philosophy, Systems General Theory and Network General Theory is used, with the purpose of developing a Quality Improvement Network allowing an optimization of systems and processes within organizations.

Key Words: Quality. Networks. Systems. Continuous improvement.

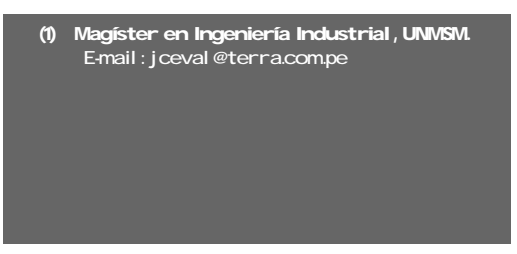

\section{INTRODUCCIÓN}

Las aplicaciones de la Teoría General de Sistemas y de la Teoría General de Redes son múltiples y se dan en diversos campos del conocimiento. En la actualidad la lucha por mejores niveles de competitividad lleva a las empresas a elevar de manera creciente su calidad y para ello es fundamental la mejora de los procesos y de los sistemas. Justamente, con ese objetivo se ha realizado el presente trabajo de investigación que ha permitido obtener una nueva herramienta de mejora de la calidad, que partiendo de las técnicas que se utilizan para la solución de los problemas de la Teoría General de Sistemas y de la Teoría General de Redes, se han combinado con el pensamiento de Mejoramiento Continuo de la calidad, obteniéndose una herramienta más poderosa que las que en la actualidad se utilizan para los trabajos de mejora de procesos y de sistemas.

Con este trabajo se apertura toda una nueva línea de desarrollo del conocimiento e investigación en el área de la calidad, que utiliza entre otros los diversos algoritmos relacionados con sistemas y redes, pero además el uso de los software y la adecuación de los mismos para el tema de la calidad. El uso de la programación lineal y programación de enteros es uno de los elementos centrales en esta nueva técnica, y con ello la posibilidad de uso de diversos programas de computación está abierta. Este trabajo muestra el primer nivel de desarrollo de esta nueva herramienta, la misma que se ha desarrollado en varios niveles de complejidad, dependiendo del tipo de problemas que se aspire resolver.

\section{FILOSOFÍA DE MEJ ORAMIENTO CONTINUO}

Deming fue uno de los primeros en plantear el mejoramiento continuo como una filosofía, y para ello propuso el ciclo de Shewart: PHVA Planear, Hacer, Verificar y Actuar. Estos cuatro pasos permiten entrar en un ciclo de mejoramiento continuo en cualquier actividad. En la actualidad se han desarrollado algunas variantes al modelo, pero en lo fundamental el modelo propuesto por Deming es el más adecuado.

En el caso de la calidad se requiere partir del nivel de calidad 1 para pasar al nivel de calidad 2, que es superior. Para dar este salto de niveles de calidad, es necesario aplicar el ciclo PHVA, que permite de manera ordenada lograr los niveles de mejora. Este "elevar la calidad" no se puede quedar en un sólo salto sino que requiere que se den una continuidad de saltos, sin límites; de allí el mejoramiento continuo.

En el nivel de calidad 1 se requiere tener una medición de la situación actual, la misma que se debe reflejar en una Norma Técnica 1, la mejora 
lleva a un nivel de calidad 2, el mismo que también requiere ser reflejado en una Norma Técnica 2; y así sucesivamente.

\section{TEORÍA GENERAL DE SISTEMAS}

La Teoría General de Sistemas, desarrollada por Bertallanffy, permite afrontar los problemas desde un enfoque distinto al tradicional. Permite ver totalidades, ver los diversos elementos que se relacionan con un problema y como es que influyen sobre su solución. El considerar las organizaciones y muchas de las realizaciones del ser humano como sistemas, lleva a considerar conjuntos de elementos que se interrelacionan entre sí y que explican su comportamiento justamente debido a dichas interrelaciones.

Cuando se trata sobre sistemas donde participa el hombre, lo más adecuado es considerar los sistemas suaves. Checkland ha desarrollado la metodología de sistemas suaves para poder estudiarlos, y llegar a solución de problemas donde participan los sistemas. Es así que se tiene su metodología de siete pasos, que son:

1. Presentación del problema.

2. Descripción espacial - modelo actual del problema.

3. Desarrollo conceptual alternativo, definición raíz.

4. Desarrollo del modelo ideal con base a la definición raíz.

5. Comparación del modelo actual con el ideal.

6. Determinación de diferencias entre el modelo actual e ideal, determinación de cambios deseables.

7. Determinación de cambios deseables y factibles, elaboración de plan de trabajo.

Esta metodología de solución de problemas permite superar el reduccionismo y ver las cosas desde un punto de vista holístico, completo; es decir, sistémico.

\section{TEORÍA GENERAL DE REDES}

La Teoría General de Redes, permite resolver diversos problemas como son el problema del transporte, el problema de asignación, el problema de transbordo, el problema de transbordo con capacidades, entre otros. Para todos ellos es de suma utilidad el uso de la programación lineal o la programación con enteros.

Es así que el modelo matemático de Red para el problema de transbordo con capacidades que se puede adecuar al caso del mejoramiento de la calidad es:

\begin{tabular}{|c|}
\hline Min. nij CijXij \\
\hline $\begin{array}{l}\text { s.a. nk Xjk }-n k X k j=L j ; j=1,2, \ldots n \\
0<=X i j<=U i j, \text { para todo }(i, j) \text { de la red }\end{array}$ \\
\hline
\end{tabular}

Donde:

Xij: denota el flujo del nodo ialj.

Lj: la oferta en el nodo j.

nij CijXij: es la función objetivo

nk Xjk: es el flujo total que sale por el nodoj.

nk Xkj: es el flujo total que entra al nodo j.

Cumpliéndose la ecuación:

Flujo total que sale del nodo j - Flujo total que entra al nodo $\mathrm{j}=$ Oferta en el nodo $\mathrm{j}$

Es de destacar que así como se considera el mínimo, también se puede presentar la situación que se esté a la búsqueda del máximo beneficio o rendimiento o productividad, y por tanto el algoritmo tendría que ajustarse de manera adecuada.

Este problema puede ser resuelto por programación lineal mediante el uso de diversos software que existen en el mercado.

\section{REDES DE MEJ ORA DE LA CALIDAD}

Para el caso de la calidad tendremos redes de diversos tipos, pero que parten de un nivel 1 y van a un nivel 2; y así sucesivamente (ver Figura 1).

Es decir el paso de un nivel 1 de calidad a un nivel 2 de calidad se realiza mediante un conjunto de acciones de mejora que en conjunto permiten lograr el objetivo, el cual se debe lograr al mínimo costo, en el mínimo tiempo y con el mínimo desperdicio; o por otro lado el máximo beneficio o el máximo rendimiento.

Las rutas que se pueden seguir para llegar de un nivel 1 a un nivel 2 son diversas, no existe una sola; sin embargo los costos son distintos y por tanto, se tienen que estudiar las rutas a fin de seleccionar la más económica.

\section{METODOLOGÍA DE REDES DE MEJ ORA DE LA CALIDAD}

Con base a los conceptos anteriores se procedió a establecer una metodología de mejora de la calidad, la misma que tiene su sustento central en la Teoría General de Redes, y es la siguiente:

Paso 1: Descripción del nivel de calidad 1. Norma Técnica 1. 


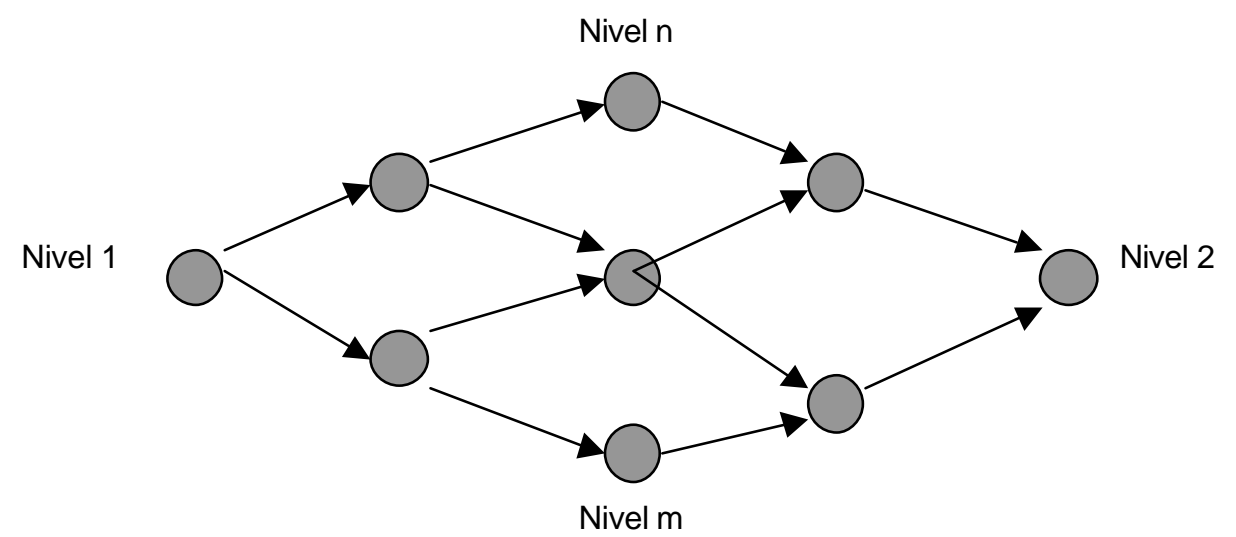

Fi g u r a 1. Red de mejora de la calidad

Paso 2: Representación sistémica: espacial del nivel de calidad 1.

Paso 3: Determinación de Definiciones Raíz del modelo actual y de un nuevo modelo ideal propuesto.

Paso 4: Representación sistémica: espacial del nivel de calidad 2, ideal.

Paso 5: Desarrollo de la Red de Transformación que permite ir del nivel de calidad 1 al nivel de calidad 2 .

Paso 6: Determinación del algoritmo que corresponde a la Red de Transformación.

Paso 7: Modelación matemática de la Red de Transformación, buscando el máximo o mínimo según corresponda.

Paso 8: Solución de la Red de Transformación, planificando las actividades que deben realizarse para lograr la transformación del nivel de calidad 1 al nivel de calidad 2 .

Paut as para I a Impl ement ación de I a Mejor a de I a Cal idad, con I as Redes de Cal idad: Caso Apl icativo

Paso 1:Un Chocolate de $58.7 \mathrm{~g}$ tiene un contenido de $10 \mathrm{mg}$ de colesterol (nivel 1 ) y se requiere modificar el proceso para que se reduzca dicho nivel a uno más bajo.

Paso 2: Representación sistémica: espacial del nivel de calidad 1 (10 mg colesterol).

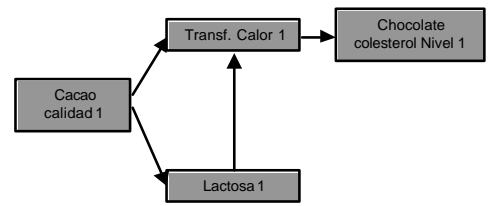

Paso 3: Se define la raíz modelo actual.

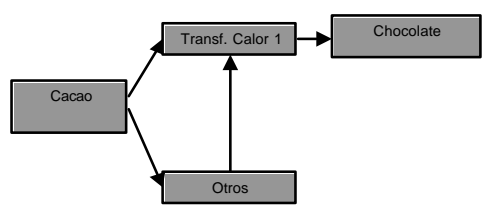

Y luego se define la raíz modelo ideal.

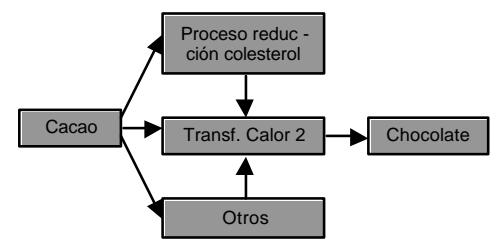

Paso 4: Representación sistémica: espacial del nivel de calidad 2, ideal (2 mg colesterol).

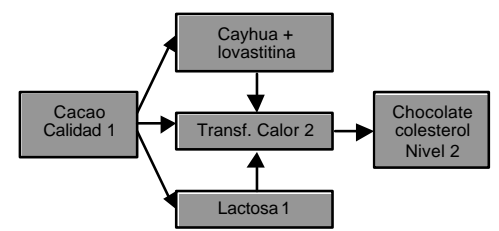

Paso 5: Desarrollo de la Red de Transformación que permite ir del nivel de calidad 1 al nivel de calidad 2 .

Nivel 2

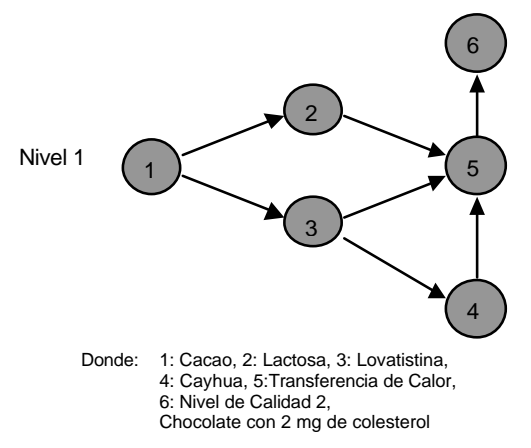


Paso 6: Determinación del algoritmo que corresponde a la Red de Transformación.

Flujo total que sale del nodo j - Flujo total que entra al nodo $\mathrm{j}=$ Oferta en el nodo $\mathrm{j}$

Nota.- En este caso el flujo transfiere tanto masa comoenergía-calor.

Paso 7: Modelación matemática de la Red de Transformación, buscando mínimo de colesterol.

\begin{tabular}{|c|}
\hline Min. nij CijXij \\
s.a. nk Xjk - nk Xkj $=L j ; j=1,2, \ldots n$ \\
$0<=X i j<=U i j$, para todo $(i, j)$ de la red.
\end{tabular}

\section{Donde:}

Xij : denota el flujo de colesterol nodo i al j.

$\mathrm{Lj}$ : la oferta de colesterol en el nodo j.

nij CijXij : es la función objetivo que busca redu-

cir la cantidad de colesterol

nk Xjk: es el flujo total de colesterol + otros que sale por el nodo j.

$\mathrm{nk} \mathrm{Xkj}$ : es el flujo total de colesterol que entra al nodo j.

Paso 8: Implica la planificación de las actividades para lograr la transformación del nivel de calidad 1 al nivel de calidad 2 .

1. Evaluación del nivel de colesterol del cacao y la lactosa.

2. Determinación del poder reductor de colesterol de la lovatistina y la cayhua.

3. Determinación de la formulación: cacao, lactosa, lovatistina y cayhua.

4. Modificación de parámetros del proceso de transferencia de calor para procesar el cacao y la lactosa.

5. Implementación del nuevo proceso para obtener chocolates y determinar sus niveles de colesterol; lo cual se deberá retroalimentar al inicio, a fin de ajustar el proceso.

Asimismo, los alcances y restricciones están definidos en las ventajas y desventajas que tiene la aplicación de los modelos de programación lineal y de optimización con enteros.

\section{CONCLUSIONES}

Se ha establecido una metodología para elevar el nivel de calidad, tanto a nivel de procesos como de sistemas.

La metodología planteada utiliza los aportes de la Teoría General de Sistemas, de la Teoría de Redes, y de la Filosofía del Mejoramiento Continuo.

La metodología de mejoramiento es de un nivel superior a las existentes a la fecha ya que no sólo se basa en análisis de tipo subjetivo, sino que usa herramientas de tipo matemático y de sistemas.

La metodología puede usar diversos software que existen en el mercado (por ejemplo Excel, Lindo, entre otros) y que se utilizan para resolver problemas de las teorías que son su fundamento.

\section{BIBLIOGRAFÍA}

1. Anderson, D. (1993). Introducción a los Modelos Cuantitativos para Administración. 6ta Edición. Editorial lberoamérica. México.

2. Checkland, P. (1993). LaMetodología de Sistemas Suaves en Acción. 1ra Edición. Editorial Prentice Hall. México.

3. Deming, E. (1986). Calidad, Productividad y Competitividad. 1ra Edición. Editorial CECSA. México.

4. Eppen, G; Gould, F.; Schmmidt, C.; Moore, J; Weatherford, L. (2000). Investigación de Operaciones en la Ciencia Administrativa. 5ta. Edición. Editorial Prentice Hall. México.

5. Harrington, H. (2000). Administración TotaldelMejoramiento Continuo. 1ra Edición. Editorial McGrawHill. Colombia.

6. Ishikawa, K. (1990). ¿Qué es el Control Total de la Calidad? 3ra Edición. Editorial Norma. Colombia.

7. Krammer. (1988). Quality Control for the Food Industry. Editorial AVI. EEUU. 